\title{
Barriers to translational research in Sjögren's syndrome with childhood onset; challenges of recognising and diagnosing an orphan rheumatic disease
}

\author{
Coziana Ciurtin, $\mathrm{PhD}^{1,2 *}$, Youna Cho, $\mathrm{MBBS}^{3}$, Muthana Al-Obaidi, $\mathrm{MD}^{4}$, \\ Elizabeth C Jury, $\mathrm{PhD}$ (Prof) ${ }^{5}$, Elizabeth J Price, $\mathrm{MD}^{6}$
}

${ }^{1}$ Centre for Adolescent Rheumatology Versus Arthritis, Department of Medicine, University College London, Rayne Building, London W1CE 6JF, U.K.

${ }^{2}$ Department of Rheumatology, University College London Hospital NHS Trust, $3^{\text {rd }}$ Floor Central, 250 Euston Road, London, NW1 2PG

${ }^{3}$ University College London Medical School, Gower Street, London WC1E 6BT

${ }^{4}$ Department of Paediatric Rheumatology, Great Ormond Hospital for Children NHS Trust, Great Ormond Street, London, WC1N 3JH

${ }^{5}$ Centre for Rheumatology, Department of Medicine, University College London, Rayne Building, London W1CE 6JF, U.K.

${ }^{6}$ Great Western Hospital NHS Foundation Trust, Swindon, SN3 6BB, UK.

*Corresponding Author:

Dr Coziana Ciurtin, PhD, FRCP, Department of Rheumatology, University College London Hospital NHS Trust, $3^{\text {rd }}$ Floor Central, 250 Euston Road, London, NW1 2PG, email: c.ciurtin@ucl.ac.uk; phone: 04402034479035. 
Keywords: Sjögren's syndrome with childhood onset, diagnosis, classification, age-related differences in clinical presentation, unmet clinical and research needs

\title{
Key messages:
}

- Sjögren's syndrome with childhood onset is an under-recognised and under-diagnosed autoimmune rheumatic disease

- Little is known about the natural history of Sjögren's syndrome with childhood onset

- The diagnosis of Sjögren's syndrome in children is delayed, because children less frequently report dryness

- There are no validated classification criteria for Sjögren's syndrome with childhood onset

- There are recognised differences in clinical presentation in childhood vs. adult onset Sjögren's syndrome; however, the long-term outcome of the childhood phenotype is not known

- There are no evidence-based treatment strategies for Sjögren's syndrome with childhood onset

\begin{abstract}
Sjögren's syndrome (SS) was considered for many years a disease of adulthood, characterised by immune infiltration of exocrine glands, leading to dryness, which is a cardinal symptom. More recently, it became apparent that although the disease in very rare in children, the clinical presentation differs from adults, posing significant challenges in recognising, diagnosing and classifying patients with SS with childhood onset. This viewpoint explores comparatively classification criteria for children (not validated) and adults with SS, as well as differences in clinical presentation in childhood vs adult onset SS, offering arguments and suggestions about how we can improve the diagnosis of SS in children. A review of the role of medical history and clinical assessment, serology, glandular function assessment and imaging, as well as
\end{abstract}


salivary and lachrymal gland biopsy in diagnosis of SS children is included, along with suggestions about further research and registry data collection required to address the unmet needs of these patients.

\section{Introduction}

Sjögren's syndrome (SS) is an autoimmune rheumatic disease (ARD) characterised by chronic lymphocytic infiltration of the exocrine glands leading to dryness, which is considered the central symptom of the disease (1). The true prevalence of SS is unclear because of various classification criteria and study methodology used, but ranging from 2.48-20.1 patients per 10,000 inhabitants according to a recent meta-analysis (2).(3). However, the variability of SS manifestations in different patients, particularly related to their disease onset within paediatric versus adult age, is reflected in the difficulty of developing universally applicable classification criteria.

SS is better described in adults. The estimated prevalence in the adult general population is 0.09 to $1.6 \%(4,5) \cdot(6) \cdot(7)$. If the disease onset is before 18 years of age, patients are described as juvenile SS or childhood SS. There are almost no epidemiological data in the paediatric population, in which the disease is considered extremely rare (8).

In approximately a third of patients, SS is associated with systemic manifestations, which more commonly include arthritis, vasculitis, anaemia and other haematological features, as well as lymphocytic interstitial pneumonia, renal tubular acidosis, cerebellar ataxia and neuropathy(9). In the adult population, SS is the ARD associated with the highest risk of lymphoma (15-20fold increase compared to the general population), with 5-10\% of SS patients developing lymphoma in their lifetime(10). The diagnosis of SS in children is often delayed, because children less frequently report dryness and commonly present with non-specific extraglandular features suggestive of other ARDs (11). The long-term outcome of SS in the paediatric population is not known and there are currently no clinical trials in this patient population.

The pathogenesis of SS is multifactorial and believed to involve an environmental trigger such as a viral infection in a genetically susceptible individual (1). Adult onset SS most commonly 
occurs in the $4^{\text {th }}$ and $5^{\text {th }}$ decades of life (12). Although rare in children and adolescents, childhood SS has gradually been recognised as a distinct clinical phenotype, with a mean age of onset between 9-12 years, according to various cohorts $(13,14)$ and characterised, as in the adult population, by a female predominance (6-7:1 in children (8) compared to 9-16:1 in adults $(15,16))$.

Several large SS patient cohorts have been established in the last few years, including the UK Primary Sjögren's syndrome registry (UKSSR), which only captures patients older than 18 years at diagnosis (17). It is important to recognise that some of the adult patients diagnosed with SS during childhood may not fulfil the classification criteria for adult onset SS when they turn 18; and therefore, they will not be captured by the existent adult registries. In addition, if these patients fulfil classification criteria later in life and are included in adult registries then, they are unlikely to be recognised as SS with childhood-onset.

Diagnosing SS accurately is challenging, as many of the clinical manifestations are commonly encountered in other ARDs, and there are no gold standard diagnostic tests. In addition, the natural course of the disease is not well characterised in children and adolescents due to the lack of large longitudinal observational studies. Therefore, expert clinical opinion remains the gold standard diagnostic criteria for childhood SS.

The aim of this viewpoint is to evaluate the existing knowledge on the clinical, serological and imaging features of childhood SS and discuss the challenges for adequate recognition and timely diagnosis of SS in children and adolescents, as well as the existent barriers to translational research. The evaluation of current treatment strategies in childhood SS is beyond the purpose of this review as none of the treatment strategies are evidence-based in this patient population.

\section{Classification criteria for Sjögren's syndrome in adults compared to children and adolescents}

In an effort to improve early recognition and diagnosis of SS in adults, 16 different classification criteria for adult onset SS have been proposed between 1965-2016(18). The American-European Consensus Group (AECG) criteria, revised in 2002 (shown in Table 1), 
have been validated in adults with SS. They include 6 domains of assessment, comprising clinical, functional, serological and histopathological criteria for SS classification (19).

More recently, Shiboski et al.(9) developed a data-driven consensus classification criteria, the 2016 ACR/EULAR criteria, which comprise 5 assessment items which are weighted differently (Table 1). To fulfil the ACR/EULAR classification criteria, a patient must score at least 4 as a sum of the weights assigned to each positive item.

A study comparing the performance of AECG and ACR/EULAR criteria has found that more patients fulfilled the ACR/EULAR ( $n=125 / 290,43.1 \%)$ than AECG classification criteria $(n=114 / 290,39.3 \%)(20)$. All patients that met the AECG criteria also met the ACR/EULAR criteria, while 11 (3.8\%) SS patients met only the ACR/EULAR criteria. In comparison to the AECG, the ACR/EULAR classification criteria offered higher sensitivity, and also captured more SS patients with early disease and systemic features.

It is increasingly recognised that SS with childhood onset can be easily underdiagnosed or misdiagnosed using the currently available classification criteria for adult SS. These criteria are not suitable for use in paediatric patients as their sensitivity is unacceptably low for this cohort of patients (21). Many case studies of juvenile SS have highlighted that sicca features are rarer in children whereas systemic and non-specific clinical manifestations are more frequent $(13,22)$.Typical serological findings such as the presence of anti-SSA/SSB and rheumatoid factor may be a late feature of disease in childhood SS and may be absent on initial presentation(8). Hence, diagnosis of SS in children can be challenging using the classification criteria validated in adults; therefore, SS with childhood onset is diagnosed based on the clinical expertise of the paediatric rheumatologist.

Bartunkova and colleagues proposed a set of criteria for the diagnosis of SS in children more than 20 years ago, but these have not been validated(23) (Table 1). Houghton and colleagues (21) compared the AECG adult criteria and the proposed pediatric criteria in a group of six patients and 128 cases found in the literature. The adult criteria were fulfilled by $14 \%$ of their cases and $39 \%$ of pediatric cases published in the literature, whereas the proposed pediatric criteria were fulfilled by $71 \%$ of the local cases and $76 \%$ of the reported pediatric cases (when compared with the gold standard of pediatric rheumatologist clinical diagnosis). The proposed paediatric classification criteria have yet to be validated (23). The Japanese Paediatric 
Sjögren's syndrome Study Group has developed new criteria for diagnosis of SS in pediatric patients (Table 1). Despite preliminary research suggesting higher sensitivity for diagnosis of SS with childhood onset, they have not been implemented widely (24).

More recently, Yokogawa et al. (22) retrospectively compared their patients with juvenile vs. adult onset SS and evaluated the applicability of existing criteria for diagnosis of juvenile onset SS. They proposed a child-specific modification which included the addition of parotitis as an objective item of salivary involvement for both the AECG (25) and Japanese criteria (26), and as an alternative to the ocular surface staining in the ACR criteria (27). They also reduced the threshold for focus score positivity to $>0$ foci $/ 4 \mathrm{~mm}^{2}$ in light of their findings on comparative histological analysis of labial salivary gland biopsies from children with SS and age matched controls (28). With these modifications, the diagnostic criteria performed better, identifying up to $85 \%$ of patients.

\section{Differences in clinical presentation in childhood vs adult onset Sjögren's syndrome}

How SS presents in an individual can vary widely in terms of both the degree of severity and the nature of the clinical presentation, variation that seems to be at least partially influenced by the age at disease onset $(29,30)$, as well as ethnicity (31). Childhood and adult SS have notable differences in the clinical manifestations. Compared to adult SS patients, the majority of studies found that paediatric SS patients had a lower incidence of sicca symptoms and presented more commonly with parotitis, systemic symptoms and extra-glandular manifestations $(13,22)$. While efforts in stratifying adult SS patients based on clinical phenotype have been met with success $(32,33)$, there are no similar studies in pediatric patients.

\section{Glandular features}

\section{Parotid gland involvement}

Recurrent parotitis or parotid enlargement has been repeatedly reported as one of the commonest presenting features in children with SS $(8,13,22)$. However, isolated parotitis is a frequent finding in childhood in the context of viral or bacterial infection, which is not clinically distinguishable from SS. Recurrence of parotitis should prompt further investigation for an underlying condition such as childhood SS. Schiffer et al.(34) reviewed twenty patients 
of 3-17 years of age who presented with recurrent parotitis. Only two patients with parotitis (10\%) met the AECG classification criteria for adult SS, while five patients (40\%) met Bartunkova's proposed criteria for juvenile SS. Only 5/20 patients eventually received a diagnosis of juvenile SS based on clinical examination and serological testing (they all fulfilled either of both AECG and Bartunkova's proposed classification criteria). This suggests that SS is a relatively common diagnosis in children and adolescents presenting with recurrent parotitis.

A multicenter survey on patients with childhood SS $(n=40)$ reported recurrent parotid swelling in $72.5 \%$ of cases (14). Drosos et al. found recurrent parotid gland enlargement in $77 \%$ of patients with childhood SS at disease onset (29). In a case series of 8 children with SS whose age at onset was 16 years or younger, parotid glad swelling was present in all patients (35). Bartunkova et al. analysed 8 children with SS and found objective signs of parotiditis in all (23). Yokogawa et al.(22) retrospectively identified that $61.5 \%$ of children with SS had features of parotitis.

Salivary gland involvement is also a common feature in adult SS and included as an assessment item in both the 2002 AECG and 2016 ACR/EULAR classification criteria. However, studies specifically reporting recurrent parotid enlargement or parotitis in adults are rare (36). A systematic review of clinical manifestations of SS in children and adult male populations reported a prevalence of parotitis that ranged between $35.7 \%-76.9 \%$, and $15.8 \%-35.7 \%$ respectively (13).

\section{Sicca symptoms and signs}

Sicca features are probably less commonly observed in paediatric patients, although still quite prevalent. A recent study of 67 patients with childhood SS found subjective ocular and oral dryness symptoms in $63 \%$ and $80 \%$, respectively (37). Ocular signs were present in $65 \%$ of children with SS (37). In this large study, $42 \%$ children with SS had a positive Schirmer test and $54 \%$ a positive ocular staining, but the sensitivity of these tests in children and adolescents with SS has not been studied. Virdee et al. (13) reported sicca symptoms in 23-80\% of children and adolescents with SS. Sicca symptoms and signs are a well-recognised feature of adultonset SS (36). They occur in more than $95 \%$ of adults with SS (38) and are included as an assessment item in both the AECG and ACR/EULAR classification criteria $(9,19)$. 


\section{Other glandular/oral manifestations}

More recently, case reports of other oral manifestations, such as ranulas (mucus extravasation cysts found on the floor of the mouth, considered to be a consequence of damage of sublingual salivary glands) in children with SS have emerged $(39,40)$. Ranulas have also been identified in adults with SS (41), raising the suspicion that this newly described clinical manifestation of SS is probably unrecognized.

\section{Extra-glandular manifestations}

Extra-glandular manifestations are usually associated with two different pathophysiological processes: lymphocytic invasion in the epithelia of organs other than the exocrine glands, e.g., interstitial nephritis or obstructive bronchiolitis; and extraepithelial involvement secondary to immune complex deposition and subsequent inflammation(1). We summarised in Table 2 the main SS studies assessing the prevalence of extra-glandular manifestations in children and adolescents.

Approximately $71 \%$ of adult SS patients present with extra-glandular manifestations (38). The lack of good quality longitudinal studies in childhood SS makes the comparison between the two clinical phenotypes difficult. Anaya et al. reviewed published studies on childhood SS as well as 5 new paediatric SS cases. Extra-glandular manifestations have been the presenting feature in $9.3 \%$ of children and adolescents, and at least one feature was present during follow up in $51 \%$ of cases, of which leukopenia was the most frequent (42). Kobayashi et al. evaluated the systemic disease activity in patients with juvenile onset-SS using the ESSDAI score, and found that a large proportion of patients had extra-articular manifestations at the disease onset as well as at peak activity, with the biological and constitutional domains of ESSDAI being the most commonly involved (43)

Constitutional symptoms, including fever, weight loss and fatigue are reported frequently in children, making the diagnosis quite challenging if present at the disease onset (8). Fatigue has been reported in 7.5-42.9\% children with SS according to different studies (Table 2), while in adults is considered one of the most common disease manifestations associated with two-fold worse fatigue scores in SS patients compared to healthy controls $(44,45)$. Yokogawa et al. (22) compared 26 childhood and 413 adult cases of SS and found that childhood SS was more frequently associated non-specific systemic manifestations, such as fever and 
lymphadenopathy. There is a lack of studies investigating the prevalence or severity of fatigue in juvenile SS.

Adult patients with SS have an increased life-time risk of lymphoma, and the most commonly occurring subtype being mucosa-associated lymphoid tissue (MALT) of the parotid glands (46). In children MALT lymphomas are extremely rare, although they have been reported (21, 47). A small case series and review of the literature comprising 5 children with SS associated with lymphoma revealed that the most common localization was within the parotid gland (3/5 cases)(47).

Arthralgia and inflammatory arthritis which have been reported in 20-50\% patients with SS, reflecting the difficulty in attributing non-specific symptoms, such as arthralgia to $\mathrm{SS}(48,49)$. Studies investigating joint involvement in SS with paediatric versus adult onset are lacking and comparisons between small cohorts of children and adults do not always match the data from larger adult studies. One paper compared 5 case reports of children with SS in addition to 34 cases of SS with childhood onset from the literature with 39 patients with SS with adult onset, and concluded that, the frequency of articular manifestations (including both arthralgia and arthritis) in children was higher than in adults (38.5\% vs. $15.4 \%)(42)$.

The most frequent skin manifestations were erythematous rashes found in more than one quarter of children with SS in some studies $(37,50)$. Muscle involvement, such as myalgia and polymyositis have also been described in a minority of patients $(23,51)$ (Table 2$)$.

Pulmonary manifestations in adults with SS are common and may be present in as many as $75 \%$ of patients (52). Interstitial lung disease (ILD) occurs in approximately $8 \%$ of adults with SS (53). The cumulative incidence of ILD in adults with SS was $10 \%$ at 1 year after diagnosis, increasing to $20 \%$ by 5 years (54). Data from children are lacking; lymphocytic interstitial pneumonitis was reported in an adolescent girl with MALT lymphoma associated with SS (55).

Renal involvement in patients with SS is rare. Interstitial nephritis, due to activated lymphocytes infiltrating the tubular epithelium, can lead to distal renal tubular acidosis (RTA), while glomerulonephritis in SS results from immune complex deposition. A large study of 715 adult SS patients reported that $4.9 \%$ of patients had clinically evident renal disease, with $49 \%$ of these having glomerulonephritis and 37\% interstitial nephritis (56). A review of 12 cases of 
RTA in children with SS and found that RTA was more frequently seen in childhood compared to adult-onset SS (57).

The spectrum of neurological manifestations associated with SS is broad and includes meningitis, myelopathy, cranial neuropathy, sensorimotor polyneuropathy, and mononeuritis multiplex (58). Isolated sensory neuropathy is considered relatively unique to SS among other ARDs and has been reported in children as well (22). A peripheral neuropathy may precede the appearance of sicca symptoms in many patients. There have been reports of optic neuropathy in pediatric-onset SS (59), as well as cases of Neuromyelitis Optica Spectrum Disorder (NMOSD), defined by a combination of optic neuritis and myelitis, exclusion of imaging features of multiple sclerosis and serological evidence of specific antibodies $(60,61)$. Other central nervous system (CNS) manifestations include hemiparesis, movement disorders, brainstem, motor neuron, and ataxic sensory neuropathy.

In conclusion, although there are no large paediatric cohort studies which can provide evidence regarding the natural history of this disease in children, the literature suggests differences in clinical manifestations and possibly a higher frequency of extra-glandular manifestations in childhood SS. The lack of longitudinal paediatric studies makes difficult to appreciate the longterm outcome patterns of SS with childhood onset.

Systemic SS manifestations are present in both children and adults, and they comprise a combination of extra-glandular manifestations affecting various organs and systems (as detailed above), frequent presence of auto-antibodies (as discussed below), as well as the presence of SS in association with other systemic autoimmune diseases, such as rheumatoid arthritis and systemic lupus erythematosus (SLE) in adults (62) and juvenile SLE and mixed connective tissue disease in children (37).

\section{How can we improve the diagnosis of SS with childhood onset and develop a translational research agenda?}




\section{Medical history and clinical assessment}

Currently, expert clinician assessment is the gold standard for diagnosis of childhood SS (55). As described above, the most common presenting feature of childhood SS is recurrent parotitis/parotid enlargement. Although not frequent, familial cases of SS have been reported (55) and some studies have shown a strong implication of HLA-DR3 in SS (63). The prevalence of SS in siblings have been estimated to be $0.09 \%$, in comparison to $0.01 \%$ in the general population (63). A diagnosis of SS in a close family member should prompt evaluation for SS patients of all ages. Longitudinal studies assessing children with SS over time are required to establish the natural history of the disease, as well as variation in disease phenotype over time, and appreciate the risk of severe complications, such as lymphoma.

\section{Serology}

Patients with SS have a variety of autoantibodies, which include rheumatoid factor (RF), antinuclear antibodies (ANA), and antibodies most commonly directed against the extractable nuclear antigens Ro/SSA and La/SSB, and others directed against organ-specific antigens such as thyroid cells or gastric mucosa. It is currently accepted that autoantibodies against Ro and La may have a pathogenic role in SS (64). In adults with SS, the prevalence of anti Ro/SSA and $\mathrm{La} / \mathrm{SSB}$ antibodies is $60-80 \%$, and $25-40 \%$, respectively (65). A systematic review of childhood SS studies found a prevalence of 36-84\% for anti-Ro/SSA and 27-65\% for antiLa/SSB autoantibodies (13). RF was positive in $36-74 \%$ of adults (66), and $27-75 \%$ children with SS (13). Other serological markers, such as auto-antibodies directed against the cytoskeletal protein $\beta$-fodrin and muscarinic receptor M3 (M3R) have been described in both children and adults with SS $(67,68)$. A pediatric SS study identified a higher prevalence of M3R antibodies in children (52.6\%) (69) compared to adults (9-14\%)(70), suggesting that these antibodies can be a valuable diagnostic marker for juvenile SS. Although the frequency of SS autoantibodies is lower in children than in adults overall, if present in children, they can be a valuable marker of subclinical SS in children with non-specific symptoms, such as fatigue (71). There are no studies to investigate differences in the disease pathogenesis potentially related to differences in the prevalence of auto-antibodies, or the role of antibodies as disease biomarkers. 


\section{Glandular function assessment and imaging}

Evaluation of salivary and lacrimal gland function is essential in determining the diagnosis. Unstimulated salivary flow test, salivary gland scintigraphy and contrast sialography have been used historically. However, the unstimulated salivary flow can be difficult to perform in younger children, and both scintigraphy and sialography are invasive procedures, involving the injection of a contrast or radioactive tracer. Ultrasonography (US), which is non-invasive, inexpensive and non-irradiating, has emerged as a possible first-line imaging tool for the diagnosis of SS. One adult SS study indicated that US performed better than both sialography and scintigraphy (72). Hammenfors et al.(37) explored the use of US to evaluate major salivary glands (parotid and submandibular glands) in childhood SS and found associations between pathologic US findings and several glandular and extra-glandular features such as hyposalivation, swollen salivary glands and autoantibodies. More recently, new imaging techniques such as magnetic resonance imaging (MRI) have been tested in SS. MRI assessment of the parotid glands has been associated with a sensitivity and specificity above $80 \%$ (73), while a comparison between US, MR imaging and MR sialography of salivary glands in aiding the diagnosis of adult SS established sensitivities of $96 \%, 81 \%$ and $78 \%$, respectively when assessed against clinical diagnosis of SS (74). The recent advances in imaging techniques and improved examiner expertise in assessing glandular changes specific for SS, support the use of non-invasive imaging techniques as first line investigations in preference to more invasive diagnostic procedures. Unfortunately, data about the sensitivity and specificity of glandular function assessment technique and salivary gland imaging in children with SS, as well as comparisons between adult and paediatric populations are lacking.

\section{Salivary and lachrymal gland biopsy}

Minor salivary gland biopsy is widely used as a part of the investigation and diagnosis of adult SS and is included in the AECG and ACR/EULAR classification criteria for SS $(9,19)$. However, it has been debated that minor salivary gland biopsy lacks sensitivity and the procedure often provides inadequate glandular tissue samples for diagnosis (75). A paediatric study reported lower sensitivity of minor salivary gland biopsy (33\%) compared to parotid gland biopsy (100\%) in a small case series of 6 children with primary and secondary SS (76). Similarly, Spijkervet et al. (77) reported that parotid gland biopsy in adults with SS is a potential alternative to minor salivary gland biopsy as the sensitivity and specificity of the two are comparable. There are no significant complications of the parotid biopsy cited in the 
literature (78), but the data are limited as this is not routine performed in the UK. Parotid gland tissue is also considered to facilitate more easily the detection of lymphoepithelial lesions and early stage of lymphoma (79), potentially having additional research benefits. Additionally, it is possible to obtain repeated biosies from the same parotid gland which is beneficial in monitoring disease progression at glandular tissue level.

Submandibular salivary gland biopsies are also associated with a good yield for diagnosis of childhood-onset SS diagnosis (Dr. Muthana Al-Obaidi, unpublished observations) and SSassociated MALT lymphoma (80).

Very few studies investigated the histopathological changes of lachrymal glands associated with SS. One adult study found that the lymphocytic focus scores were significantly higher in lacrimal gland biopsies compared to labial salivary gland biopsies. Furthermore, accessing both the lacrimal gland and labial salivary glands was associated with a significantly greater yield for detection of abnormalities specific for SS and facilitated diagnosis more than using either option alone (81).

\section{Conclusion}

Childhood onset SS is an orphan ARD which requires a huge effort from the rheumatology community to improve its recognition and timely diagnosis. Differences in the clinical presentation and natural course of the disease exist between childhood and adult-onset SS, therefore using the same classification criteria for both disease phenotypes is inadequate. The main challenges for recognising and diagnosing SS in childhood onset are summarised in Figure 1. One of the main limitations in interpreting the literature data related to SS with childhood onset is related to the lack of good quality, large cohort studies. Further research is required to improve our knowledge about the natural course of SS with childhood onset, including the life-long risk of lymphoma, as well as investigate the accuracy and usefulness of adult-SS tailored investigations for this patient group to enhance recognition and subsequently improve management.

Although many investigations have been useful in aiding the diagnosis of adult-onset SS, there is a need for future research to establish age-adjusted cut-off values for objective measurements of exocrine gland secretion (e.g. unstimulated/stimulated salivary flow, Schirmer's test, ocular staining scores) before they can be implemented in children. In addition, imaging studies should evaluate the sensitivity and specificity of salivary gland imaging (weather US, MRI or MR sialography) for SS with childhood onset, as well as their role in aiding the timely diagnosis 
of SS in children. Novel technologies, including saliva or tear biomarkers, corneal impression cytology, tear osmolarity should also be evaluated to establish their clinical utility in diagnosis and monitoring of SS with childhood onset.

Despite efforts from SHARE (Single Hub and Access point for paediatric Rheumatology in Europe) to optimise and disseminate diagnostic and management strategies in Europe for children and young adults with rheumatic diseases, as well as CARRA (Childhood Arthritis and Rheumatology Research Alliance) in the US to support registry data collection across all paediatric rheumatic diseases, more support is required to address the unmet needs of children with SS. We initiated in 2020 the first multi-centre cohort study of SS with childhood onset in the UK - the PaedSSCoRe (Paediatric Sjögren syndrome Cohort study and Repository), which will start recruiting patients by the end of the year.

The translational research agenda in this rare ARD should address differences in genetic background, ethiopathogenesis, clinical presentation, serological and imaging biomarkers, as well as validate classification criteria and disease outcome measures tailored to this disease population. We identified barriers and unmet needs for patients with SS with childhood onset and propose a translational research agenda to address them (Figure 2). Although, assessment of treatment and management strategies for children with SS was beyond the scope of this review, we support the inclusion of patients with SS with childhood onset in national and international registries linking paediatric and adult rheumatology specialists, to enable evaluation of the natural history of this disease through collection of longitudinal data which will inform further research agenda and clinician training focused on improving the recognition and classification of this rare disease phenotype for ultimate patient benefit.

\section{Search strategy and selection criteria}

This is a narrative viewpoint based on literature searches (PubMed from January 1950 to April 2020) for systematic reviews, studies, case series and case-reports of patients with SS with childhood onset using the following MeSH terms: juvenile Sjögren's syndrome OR Sjögren's syndrome with childhood OR juvenile OR paediatric onset OR Sjögren's syndrome in children OR Sjögren's syndrome in paediatric population. Reports including more than 5 cases were described in detail and included in a table describing the prevalence of extra-glandular 
manifestations, while case reports of less than 5 patients have been discussed in the text if relevant for describing rarer disease manifestations. Papers published in another language than English have been excluded. This review is not intended to be comprehensive, but rather focused on challenges in recognising and classifying SS with childhood onset. In addition, this viewpoint highlights the barriers to translational research in this rare disease and proposes strategies to overcome them.

\section{References:}

1. Nocturne G, Mariette X. Advances in understanding the pathogenesis of primary Sjogren's syndrome. Nat Rev Rheumatol. 2013;9(9):544-56.

2. Qin B, Wang J, Yang Z, Yang M, Ma N, Huang F, et al. Epidemiology of primary Sjogren's syndrome: a systematic review and meta-analysis. Ann Rheum Dis. 2015;74(11):1983-9.

3. Cornec D, Chiche L. Is primary Sjogren's syndrome an orphan disease? A critical appraisal of prevalence studies in Europe. Ann Rheum Dis. 2015;74(3):e25.

4. Anagnostopoulos I, Zinzaras E, Alexiou I, Papathanasiou AA, Davas E, Koutroumpas A, et al. The prevalence of rheumatic diseases in central Greece: a population survey. BMC Musculoskelet Disord. 2010;11:98.

5. Dafni UG, Tzioufas AG, Staikos P, Skopouli FN, Moutsopoulos HM. Prevalence of Sjogren's syndrome in a closed rural community. Ann Rheum Dis. 1997;56(9):521-5.

6. Bowman SJ, Ibrahim GH, Holmes G, Hamburger J, Ainsworth JR. Estimating the prevalence among Caucasian women of primary Sjogren's syndrome in two general practices in Birmingham, UK. Scandinavian journal of rheumatology. 2004;33(1):39-43.

7. Tomsic M, Logar D, Grmek M, Perkovic T, Kveder T. Prevalence of Sjogren's syndrome in Slovenia. Rheumatology (Oxford). 1999;38(2):164-70.

8. de Souza TR, Silva IH, Carvalho AT, Gomes VB, Duarte AP, Leao JC, et al. Juvenile Sjogren syndrome: distinctive age, unique findings. Pediatr Dent. 2012;34(5):427-30.

9. Shiboski CH, Shiboski SC, Seror R, Criswell LA, Labetoulle M, Lietman TM, et al. 2016 American College of Rheumatology/European League Against Rheumatism classification criteria for primary Sjogren's syndrome: A consensus and data-driven methodology involving three international patient cohorts. Ann Rheum Dis. 2017;76(1):9-16. 10. Anderson LA, Gadalla S, Morton LM, Landgren O, Pfeiffer R, Warren JL, et al. Population-based study of autoimmune conditions and the risk of specific lymphoid malignancies. Int J Cancer. 2009;125(2):398-405.

11. Singer NG, Tomanova-Soltys I, Lowe R. Sjogren's syndrome in childhood. Curr Rheumatol Rep. 2008;10(2):147-55.

12. Ramirez Sepulveda JI, Kvarnstrom M, Brauner S, Baldini C, Wahren-Herlenius M. Difference in clinical presentation between women and men in incident primary Sjogren's syndrome. Biol Sex Differ. 2017;8:16.

13. Virdee S, Greenan-Barrett J, Ciurtin C. A systematic review of primary Sjogren's syndrome in male and paediatric populations. Clinical rheumatology. 2017;36(10):2225-36. 
14. Cimaz R, Casadei A, Rose C, Bartunkova J, Sediva A, Falcini F, et al. Primary Sjogren syndrome in the paediatric age: a multicentre survey. Eur J Pediatr. 2003;162(10):661-5.

15. Hayter SM, Cook MC. Updated assessment of the prevalence, spectrum and case definition of autoimmune disease. Autoimmun Rev. 2012;11(10):754-65.

16. Maciel G, Crowson CS, Matteson EL, Cornec D. Prevalence of Primary Sjogren's Syndrome in a US Population-Based Cohort. Arthritis Care Res (Hoboken).

2017;69(10):1612-6.

17. Ng WF, Bowman SJ, Griffiths B, group Us. United Kingdom Primary Sjogren's Syndrome Registry--a united effort to tackle an orphan rheumatic disease. Rheumatology (Oxford). 2011;50(1):32-9.

18. Billings M, Amin Hadavand M, Alevizos I. Comparative analysis of the 2016 ACREULAR and the 2002 AECG classification criteria for Sjogren's syndrome: Findings from the NIH cohort. Oral diseases. 2018;24(1-2):184-90.

19. Vitali C, Bombardieri S, Jonsson R, Moutsopoulos HM, Alexander EL, Carsons SE, et al. Classification criteria for Sjogren's syndrome: a revised version of the European criteria proposed by the American-European Consensus Group. Ann Rheum Dis. 2002;61(6):554-8.

20. Le Goff M, Cornec D, Jousse-Joulin S, Guellec D, Costa S, Marhadour T, et al. Comparison of 2002 AECG and 2016 ACR/EULAR classification criteria and added value of salivary gland ultrasonography in a patient cohort with suspected primary Sjogren's syndrome. Arthritis Res Ther. 2017;19(1):269.

21. Houghton K, Malleson P, Cabral D, Petty R, Tucker L. Primary Sjogren's syndrome in children and adolescents: are proposed diagnostic criteria applicable? J Rheumatol. 2005;32(11):2225-32.

22. Yokogawa N, Lieberman SM, Sherry DD, Vivino FB. Features of childhood Sjogren's syndrome in comparison to adult Sjogren's syndrome: considerations in establishing child-specific diagnostic criteria. Clin Exp Rheumatol. 2016;34(2):343-51.

23. Bartunkova J, Sediva A, Vencovsky J, Tesar V. Primary Sjogren's syndrome in children and adolescents: proposal for diagnostic criteria. Clin Exp Rheumatol. 1999;17(3):381-6.

24. Tomiita M KI, Inoue Y, Okamoto N, Iwata N, Nonaka Y, Hara R, Umebayashi H, Itoh Y, Mori M. . Validation of New Criteria in "The Guidance for Diagnosis of Sjögren's Syndrome in Pediatric Patients" [abstract]. . Arthritis Rheumatol 2017; 69 (suppl 10). 2017. 25. Vitali C BS, Jonsson R, Moutsopoulos HM, Alexander EL, Carsons SE, Daniels TE, Fox PC, Fox RI, Kassan SS, Pillemer SR, Talal N, Weisman MH; European Study Group on Classification Criteria for Sjögren's Syndrome. Classification criteria for Sjögren's syndrome: a revised version of the European criteria proposed by the American-European Consensus Group. Ann Rheum Dis. 2002;61(6):554-8.

26. Fujibayashi T, Sugai S, Miyasaka N, Hayashi Y, Tsubota K. Revised Japanese criteria for Sjogren's syndrome (1999): availability and validity. Modern rheumatology / the Japan Rheumatism Association. 2004;14(6):425-34.

27. Shiboski SC, Shiboski CH, Criswell L, Baer A, Challacombe S, Lanfranchi H, et al. American College of Rheumatology classification criteria for Sjogren's syndrome: a datadriven, expert consensus approach in the Sjogren's International Collaborative Clinical Alliance cohort. Arthritis care \& research. 2012;64(4):475-87.

28. Yokogawa N, Lieberman SM, Alawi F, Bout-Tabaku S, Guttenberg M, Sherry DD, et al. Comparison of labial minor salivary gland biopsies from childhood Sjogren syndrome and age-matched controls. The Journal of rheumatology. 2014;41(6):1178-82. 
29. Drosos AA, Tsiakou EK, Tsifetaki N, Politi EN, Siamopoulou-Mavridou A.

Subgroups of primary Sjogren's syndrome. Sjogren's syndrome in male and paediatric Greek patients. Ann Rheum Dis. 1997;56(5):333-5.

30. Botsios C, Furlan A, Ostuni P, Sfriso P, Andretta M, Ometto F, et al. Elderly onset of primary Sjogren's syndrome: clinical manifestations, serological features and oral/ocular diagnostic tests. Comparison with adult and young onset of the disease in a cohort of 336 Italian patients. Joint Bone Spine. 2011;78(2):171-4.

31. Lin DF, Yan SM, Zhao Y, Zhang W, Li MT, Zeng XF, et al. Clinical and prognostic characteristics of 573 cases of primary Sjogren's syndrome. Chin Med J (Engl). 2010;123(22):3252-7.

32. Tarn JF H-TN, Lendrem DV, Mariette X, Saraux A, Devauchelle-Pensec V, et al. Symptom-based stratification of patients with primary Sjögren's syndrome: multidimensional characterisation of international observational cohorts and reanalyses of randomised clinical trials. The Lancet Rheumatology, Volume 1, Issue 2, e85 - e94. 2019. 33. Noaiseh G, Baer AN. Toward better outcomes in Sjogren's syndrome: The promise of a stratified medicine approach. Best Pract Res Clin Rheumatol. 2020:101475.

34. Schiffer BL, Stern SM, Park AH. Sjogren's syndrome in children with recurrent parotitis. Int J Pediatr Otorhinolaryngol. 2020;129:109768.

35. Chudwin DS, Daniels TE, Wara DW, Ammann AJ, Barrett DJ, Whitcher JP, et al. Spectrum of Sjogren syndrome in children. J Pediatr. 1981;98(2):213-7.

36. Kassan SS, Moutsopoulos HM. Clinical manifestations and early diagnosis of Sjogren syndrome. Arch Intern Med. 2004;164(12):1275-84.

37. Hammenfors DS, Valim V, Bica B, Pasoto SG, Lilleby V, Nieto-Gonzalez JC, et al. Juvenile Sjogren's Syndrome: Clinical Characteristics With Focus on Salivary Gland Ultrasonography. Arthritis Care Res (Hoboken). 2020;72(1):78-87.

38. Ramos-Casals M, Solans R, Rosas J, Camps MT, Gil A, Del Pino-Montes J, et al. Primary Sjogren syndrome in Spain: clinical and immunologic expression in 1010 patients. Medicine (Baltimore). 2008;87(4):210-9.

39. Means C, Aldape MA, King E. Pediatric primary Sjogren syndrome presenting with bilateral ranulas: A case report and systematic review of the literature. Int J Pediatr Otorhi. 2017;101:11-9.

40. Lieberman SM, Lu A, McGill MM. Oral lesions as presenting feature of childhood Sjogren syndrome. Int J Pediatr Otorhi. 2018;113:303-4.

41. Sato K, Yoshida Y, Sakai K, Shibui T, Hashimoto K, Baba A, et al. Sjogren's syndrome and ranula development. Oral Dis. 2019;25(6):1664-7.

42. Anaya JM, Ogawa N, Talal N. Sjogren's syndrome in childhood. J Rheumatol. 1995;22(6):1152-8.

43. Kobayashi I, Okura Y, Ueki M, Tozawa Y, Takezaki S, Yamada M, et al. Evaluation of systemic activity of pediatric primary Sjogren's syndrome by EULAR Sjogren's syndrome disease activity index (ESSDAI). Mod Rheumatol. 2019;29(1):130-3.

44. Segal B, Thomas W, Rogers T, Leon JM, Hughes P, Patel D, et al. Prevalence, severity, and predictors of fatigue in subjects with primary Sjogren's syndrome. Arthritis Rheum. 2008;59(12):1780-7.

45. Barendregt PJ, Visser MR, Smets EM, Tulen JH, van den Meiracker AH, Boomsma F, et al. Fatigue in primary Sjogren's syndrome. Ann Rheum Dis. 1998;57(5):291-5.

46. Fallah M, Liu X, Ji J, Forsti A, Sundquist K, Hemminki K. Autoimmune diseases associated with non-Hodgkin lymphoma: a nationwide cohort study. Ann Oncol.

2014;25(10):2025-30. 
47. Tesher MS, Esteban Y, Henderson TO, Villanueva G, Onel KB. Mucosal-associated Lymphoid Tissue (MALT) Lymphoma in Association With Pediatric Primary Sjogren Syndrome: 2 Cases and Review. J Pediatr Hematol Oncol. 2019;41(5):413-6.

48. Venables PJ. Management of patients presenting with Sjogren's syndrome. Best Pract Res Clin Rheumatol. 2006;20(4):791-807.

49. Atzeni F, Sarzi-Puttini P, Lama N, Bonacci E, Bobbio-Pallavicini F, Montecucco C, et al. Anti-cyclic citrullinated peptide antibodies in primary Sjogren syndrome may be associated with non-erosive synovitis. Arthritis Res Ther. 2008;10(3):R51.

50. Tomiita M, Saito K, Kohno Y, Shimojo N, Fujikawa S, Niimi H. The clinical features of Sjögren's syndrome in Japanese children. Acta paediatrica Japonica : Overseas edition. 1997;39(2):268-72.

51. Schuetz C, Prieur AM, Quartier P. Sicca syndrome and salivary gland infiltration in children with autoimmune disorders: when can we diagnose Sjogren syndrome? Clin Exp Rheumatol. 2010;28(3):434-9.

52. Parke AL. Pulmonary manifestations of primary Sjogren's syndrome. Rheum Dis Clin North Am. 2008;34(4):907-20, viii.

53. Ito I, Nagai S, Kitaichi M, Nicholson AG, Johkoh T, Noma S, et al. Pulmonary manifestations of primary Sjogren's syndrome: a clinical, radiologic, and pathologic study. American journal of respiratory and critical care medicine. 2005;171(6):632-8.

54. Nannini C, Jebakumar AJ, Crowson CS, Ryu JH, Matteson EL. Primary Sjogren's syndrome 1976-2005 and associated interstitial lung disease: a population-based study of incidence and mortality. BMJ Open. 2013;3(11):e003569.

55. Houghton KM, Cabral DA, Petty RE, Tucker LB. Primary Sjogren's syndrome in dizygotic adolescent twins: one case with lymphocytic interstitial pneumonia. J Rheumatol. 2005;32(8):1603-6.

56. Goules AV, Tatouli IP, Moutsopoulos HM, Tzioufas AG. Clinically significant renal involvement in primary Sjogren's syndrome: clinical presentation and outcome. Arthritis Rheum. 2013;65(11):2945-53.

57. Pessler F, Emery H, Dai L, Wu YM, Monash B, Cron RQ, et al. The spectrum of renal tubular acidosis in paediatric Sjogren syndrome. Rheumatology (Oxford). 2006;45(1):85-91.

58. Segal B, Carpenter A, Walk D. Involvement of nervous system pathways in primary Sjogren's syndrome. Rheum Dis Clin North Am. 2008;34(4):885-906, viii.

59. Berman JL, Kashii S, Trachtman MS, Burde RM. Optic neuropathy and central nervous system disease secondary to Sjogren's syndrome in a child. Ophthalmology. 1990;97(12):1606-9.

60. Kornitzer JM, Kimura Y, Janow G. Pediatric Neuromyelitis Optica Spectrum Disorder and Sjogren Syndrome: More Common Than Previously Thought? Reply. J Rheumatol. 2017;44(6):960-.

61. Gmuca S, Lieberman SM, Mfhta J. Pediatric Neuromyelitis Optica Spectrum Disorder and Sjogren Syndrome: More Common Than Previously Thought? J Rheumatol. 2017;44(6):959-+.

62. Malladi AS, Sack KE, Shiboski SC, Shiboski CH, Baer AN, Banushree R, et al. Primary Sjogren's syndrome as a systemic disease: a study of participants enrolled in an international Sjogren's syndrome registry. Arthritis Care Res (Hoboken). 2012;64(6):911-8. 63. Cobb BL, Lessard CJ, Harley JB, Moser KL. Genes and Sjogren's syndrome. Rheum Dis Clin North Am. 2008;34(4):847-68, vii.

64. Routsias JG, Tzioufas AG. Sjogren's syndrome--study of autoantigens and autoantibodies. Clin Rev Allergy Immunol. 2007;32(3):238-51. 
65. Tzioufas AG, Tatouli IP, Moutsopoulos HM. Autoantibodies in Sjogren's syndrome: clinical presentation and regulatory mechanisms. Presse Med. 2012;41(9 Pt 2):e451-60. 66. Bournia VK, Vlachoyiannopoulos PG. Subgroups of Sjogren syndrome patients according to serological profiles. J Autoimmun. 2012;39(1-2):15-26.

67. Chen Q, Li X, He W, Zhang H, Gao A, Cheng Y, et al. The epitope study of alphafodrin autoantibody in primary Sjogren's syndrome. Clin Exp Immunol. 2007;149(3):497503.

68. Maeno N, Takei S, Imanaka H, Oda H, Yanagi K, Hayashi Y, et al. Anti-alpha-fodrin antibodies in Sjogren's syndrome in children. The Journal of rheumatology. 2001;28(4):8604.

69. Nakamura Y, Wakamatsu E, Matsumoto I, Tomiita M, Kohno Y, Mori M, et al. High prevalence of autoantibodies to muscarinic-3 acetylcholine receptor in patients with juvenileonset Sjogren syndrome. Annals of the Rheumatic Diseases. 2008;67(1):136-7.

70. Naito Y, Matsumoto I, Wakamatsu E, Goto D, Sugiyama T, Matsumura R, et al. Muscarinic acetylcholine receptor autoantibodies in patients with Sjogren's syndrome. Annals of the Rheumatic Diseases. 2005;64(3):510-1.

71. Itoh Y, Imai T, Fujino O, Igarashi T, Fukunaga Y. Subclinical Sjogren's syndrome and anti-Ro/SSA-positive autoimmune fatigue syndrome in children. Mod Rheumatol. 2002;12(3):201-5.

72. Salaffi F, Carotti M, Iagnocco A, Luccioli F, Ramonda R, Sabatini E, et al. Ultrasonography of salivary glands in primary Sjogren's syndrome: a comparison with contrast sialography and scintigraphy. Rheumatology (Oxford). 2008;47(8):1244-9.

73. Kojima I, Sakamoto M, Iikubo M, Kumamoto H, Muroi A, Sugawara Y, et al. Diagnostic performance of MR imaging of three major salivary glands for Sjogren's syndrome. Oral Dis. 2017;23(1):84-90.

74. Niemela RK, Takalo R, Paakko E, Suramo I, Paivansalo M, Salo T, et al. Ultrasonography of salivary glands in primary Sjogren's syndrome. A comparison with magnetic resonance imaging and magnetic resonance sialography of parotid glands. Rheumatology (Oxford). 2004;43(7):875-9.

75. Vitali C, Tavoni A, Simi U, Marchetti G, Vigorito P, d'Ascanio A, et al. Parotid sialography and minor salivary gland biopsy in the diagnosis of Sjogren's syndrome. A comparative study of 84 patients. J Rheumatol. 1988;15(2):262-7.

76. McGuirt WF, Jr., Whang C, Moreland W. The role of parotid biopsy in the diagnosis of pediatric Sjogren syndrome. Arch Otolaryngol Head Neck Surg. 2002;128(11):1279-81.

77. Spijkervet FK, Haacke E, Kroese FG, Bootsma H, Vissink A. Parotid Gland Biopsy, the Alternative Way to Diagnose Sjogren Syndrome. Rheum Dis Clin North Am.

2016;42(3):485-99.

78. Pijpe J, Kalk WW, van der Wal JE, Vissink A, Kluin PM, Roodenburg JL, et al. Parotid gland biopsy compared with labial biopsy in the diagnosis of patients with primary Sjogren's syndrome. Rheumatology (Oxford). 2007;46(2):335-41.

79. Hu S, Zhou M, Jiang J, Wang J, Elashoff D, Gorr S, et al. Systems biology analysis of Sjogren's syndrome and mucosa-associated lymphoid tissue lymphoma in parotid glands. Arthritis Rheum. 2009;60(1):81-92.

80. Movahed R, Weiss A, Velez I, Dym H. Submandibular gland MALT lymphoma associated with Sjogren's syndrome: case report. J Oral Maxillofac Surg. 2011;69(11):29249.

81. Xu KP, Katagiri S, Takeuchi T, Tsubota K. Biopsy of labial salivary glands and lacrimal glands in the diagnosis of Sjogren's syndrome. J Rheumatol. 1996;23(1):76-82. 
Authors statements: $\mathrm{CC}$ drafted the manuscript, tables and figures. YC and $\mathrm{CC}$ reviewed the literature data. All authors contributed to, reviewed and approved the final manuscript as submitted.

Declaration of interest: All authors declared no conflicts of interest.

Funding: This project received no funding. CC and ECJ are supported by NIHR UCLH Biomedical Research Centre grants (BRC772/III/EJ/101350 and BRC525/III/CC/191350). This work was performed within the Centre for Adolescent Rheumatology Versus Arthritis at UCL UCLH and GOSH supported by grants from Versus Arthritis (21593 and 20164), GOSCC, and the NIHR-Biomedical Research Centres at both GOSH and UCLH. The views expressed are those of the authors and not necessarily those of the NHS, the NIHR or the Department of Health. 